JEEP 2011, $00002(2011)$

DOI: $10.1051 /$ jeep/201100002

(c) Owned by the authors, published by EDP Sciences, 2011

\title{
Crystal growth in condensed phase and phase diagrams
}

\author{
C. Goutaudier
}

Université de Lyon, Université Claude Bernard Lyon 1, Laboratoire Multimatériaux et Interfaces - UMR CNRS 5615, 69622 Villeurbanne cedex, France

christelle.goutaudier@univ-lyon1.fr

\begin{abstract}
The crystal growth techniques used in industry usually take place in melt and more than $80 \%$ of the crystals produced have a congruent melting composition. However, the complexity of single-crystal matrices is constantly increasing ( 4 constituents or even more) and in these multi-constituent systems, very few are fully stoichiometric compounds with a congruent melting composition. Whatever the technique used, to master the growth process, a required first step is to know the stability domain of the solid and liquid phases concerned in a temperature-pressure-composition coordinate system. The choice of growth technique to be implemented will depend primarily on the thermodynamic parameters and the nucleation conditions which will result in crystal generation.
\end{abstract}

\section{Introduction}

The term "krystallos" appears in antiquity and comes from the Greek word cryo which designates ice. A crystal is a solid material which represents on the one hand an external symmetry delineated by a set of crystalline surfaces, on the other hand an internal symmetry resulting from the three-dimensional layout of entities according to a regular model called the lattice system. A monocrystalline solid is ideally made of a single crystal, for which the spatial arrangement is a regular and periodic repetition of a large number of atoms, molecules or ions. The single crystal may be considered as the "perfect crystal", and its relevance lies in its great structural homogeneity, i.e. the environment of each similar entity is exactly the same. It is essential to the study and utilisation of the solid-state physical properties which depend on the structure (mechanical, electromagnetic, acoustic properties, etc.). Single crystals are at the root of a number of high-technology applications in such fields as electronics, optics, radiation detection, etc. The industrial production of bulk single crystals started in the 1990s and has developed continuously to reach about ten thousand tons today [1]. A few examples of crystals as well as the distribution of industrial production among the various application fields are presented in Table 1. The microelectronics and semiconductors industries constitute the major part and this is constantly increasing.
The fundamental aspects of crystal growing were deduced from the first crystallisation experiments at the beginning of the $18^{\text {th }}$ century, then improved with the development of thermodynamics (late $19^{\text {th }}$ century) and finally with the theories of nucleation/growth and the role of transport properties [2]. The choice of crystal growth technique to be implemented will result from a compromise between:

- on the one hand, the main four growth parameters, i.e. growth temperature, supersaturation, temperature gradient and fluid phase dynamics;

- on the other hand, the crystal qualities imposed by the technological application and the economic factor: stoichiometry, impurity rate, homogeneity of composition, inclusions, structural perfection, rapidity and profitability.

The purpose of this paper is to address crystal growth through a thermodynamic approach, and more specifically the choice of methodology to be implemented and the control of crystal composition from the knowledge of equilibria between phases. 
Table 1. A few industrial single crystal examples by application field.

\begin{tabular}{|c|c|c|}
\hline $\begin{array}{c}\text { Application } \\
\text { field }\end{array}$ & $\begin{array}{c}\text { Examples of } \\
\text { crystals }\end{array}$ & $\begin{array}{c}\text { Distribution } \\
\text { of industrial } \\
\text { production }\end{array}$ \\
\hline Semiconductors
\end{tabular}

\section{Thermodynamics}

A crystal is formed through ions, atoms or molecules going from a disordered state to an ordered state. The disordered "mother" phase may be a gas, a liquid or even a solid. It will initially be maintained in thermodynamic equilibrium but this equilibrium must be disturbed to result in the phase-transition process.

\subsection{Phase relation}

In order to make massive single crystals, the crystal growth methods most widely used industrially usually call for a liquid-solid transition. The vapour phase techniques are essentially kept for thin layer growth. In any case, to master the growth process, a required first step is to know the stability domain of the solid and liquid (or gas) phases concerned in a temperaturecomposition or even temperature-pressure-composition diagram.

\subsubsection{The case of unary systems}

The existence domain of solid-liquid-gas phases of a unary system is conventionally represented in a P-T diagram and the simplest cases include 3 phases. The relationships between each of the phases are described by Clapeyron's relation which, in the case of solid-liquid equilibrium, is expressed by:

$d P=\frac{\Delta H_{\text {fus }}}{\Delta V_{\text {fus }}} d \operatorname{LnT}$

where $\Delta H_{f u s}$ is the enthalpy variation during the transition phase, considered here as independent of temperature, and $\Delta V_{f u s}$ is the molar volume variation. It is then easy to define the temperature domain which is favourable to crystal growth.
The most complex cases appear when a fourth phase is observed, i.e. two solid phases that correspond to two allotropic varieties of the pure constituent. There are two types. Either the structural transition occurs during a change in temperature (Figure 1a): there is only one solid-liquid equilibrium curve and it will be impossible to crystallise the low temperature variety from the liquid phase. Or the structural transition is linked to a change in pressure (Figure 1b): there is a solid-liquid equilibrium curve for each crystal variety and different pressures should be used to crystallise one or the other; this is the case, for instance, of carbon (graphite or diamond).

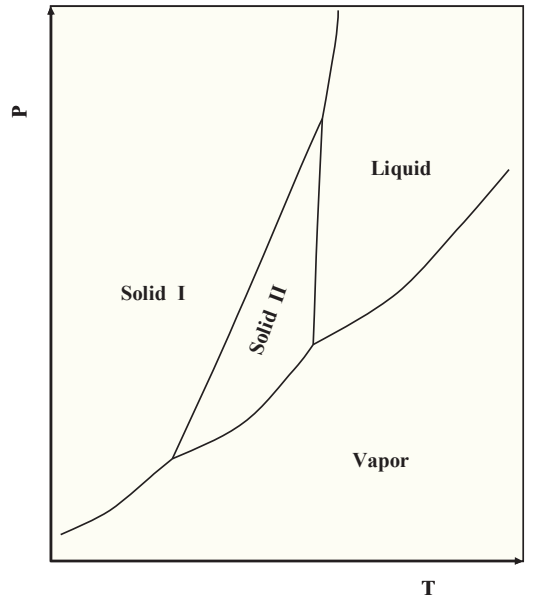

(a)

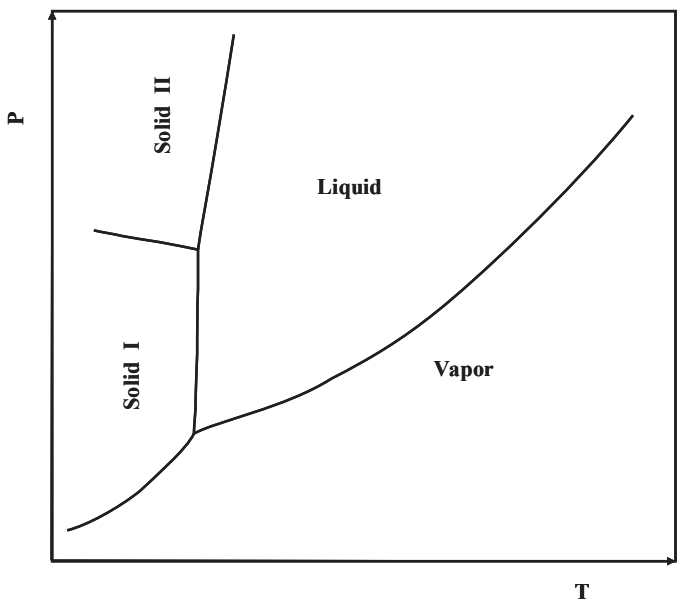

(b)

Fig. 1. Existence domain of four phases in a unary system. (a) enantiotropic case (b) monotropic case

\subsubsection{The case of binary systems}

When the crystal to be made consists of two independent constituents, it is easy to refer to temperaturecomposition isobaric representations in order to guide the choice of crystal growth technique.

Therefore the solid-liquid equilibrium curves are represented for each constituent $i$ by the relation:

$d \operatorname{Ln} \frac{a_{i, s o l}}{a_{i, l i q}}=\frac{\Delta H_{i, f u s}}{R T^{2}} d T$ 
where $a_{i}$ is the activity of constituent $i$ in the binary mixture, and $\Delta H_{i, f u s}$ is its melting enthalpy under the pressure considered.

The simplest case of solid-liquid equilibria utilisation will be for compounds with a congruent melting composition, i.e. compounds for which the melted liquid composition is identical to the composition of the solid from which it comes (Figure 2a). The principle of crystalgrowing in melt will consist in slowly crystallising the material from its liquid phase, at the equilibrium melting temperature. Most of the massive stoichiometric crystals industrially fabricated have a congruent melting composition.

However, the melting equilibrium may not be processable sometimes. This is the case of:

- compounds with a more or less extended solid solution domain (Figure 2b). Controlling the solid composition during cooling is tricky and will result in high inhomogeneities in the single crystal,

- compounds which break down by a peritectic reaction (Figure 2c). The composition of the melted liquid may be very different from that expected for the solid phase,

- compounds presenting several structure varieties (figure 2d). Cooling a single crystal made in its high temperature form will result in its destruction upon going through the transition isotherm on the way to the low temperature phase.

In these specific cases, the techniques implemented will consist in processing a solid-liquid equilibrium for which the liquid phase has a different composition from that of the desired solid: this will be called solution or flux growth.

\subsubsection{The case of higher order systems}

The technological advances made in the last few decades imply that the complexity of single-crystal matrices is constantly increasing (4 constituents or even more), so that the homogeneity of the material's chemical composition conditions its process through the structure/property relationship. In these multi-constituent systems, very few are fully stoichiometric compounds with a congruent melting composition. The general case looks more like an accumulation of all abnormalities described in the case of binary compounds (solid solution, decomposition, crystal varieties). Only the techniques starting from an initial composition flux different from that of the crystal will lead to crystal growth in the desired phase. This type of method inevitably requires knowledge of the solid-liquid equilibria in the ternary or even higher-order systems. As an example, we can cite the $\mathrm{KY}\left(\mathrm{WO}_{4}\right)_{2}$ compound for which the single crystals are studied for their civilian laser applications. Study of the existence domain of $\mathrm{KY}\left(\mathrm{WO}_{4}\right)_{2}$ in the ternary system $\mathrm{K}_{2} \mathrm{O}-\mathrm{Y}_{2} \mathrm{O}_{3}-\mathrm{WO}_{3}$ demonstrated that:

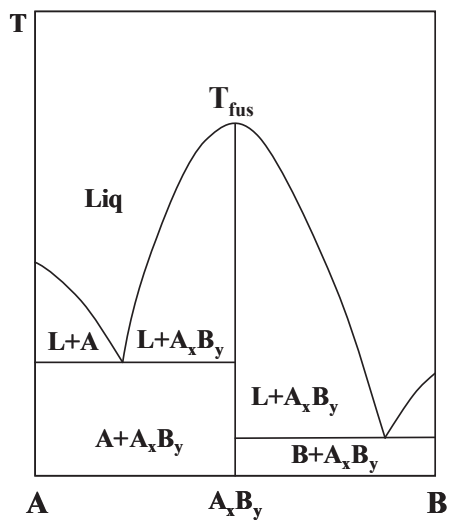

(a)

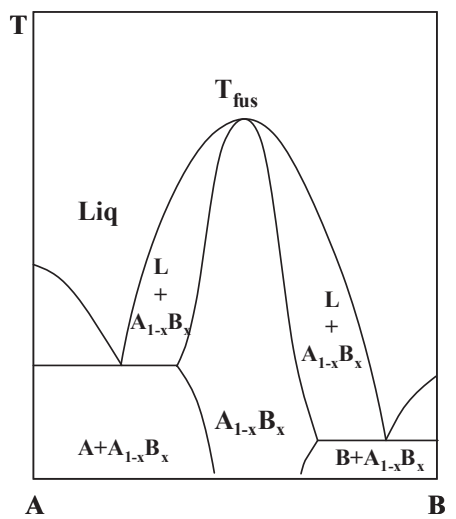

(b)

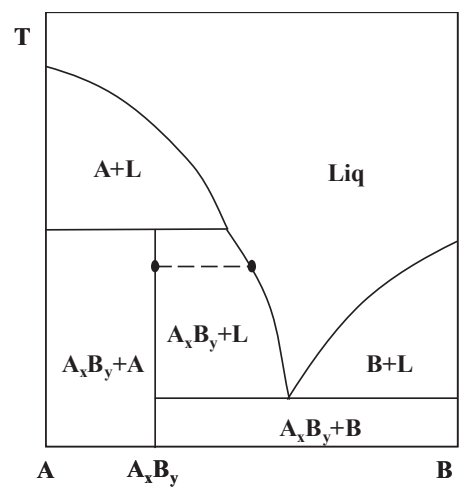

(c)

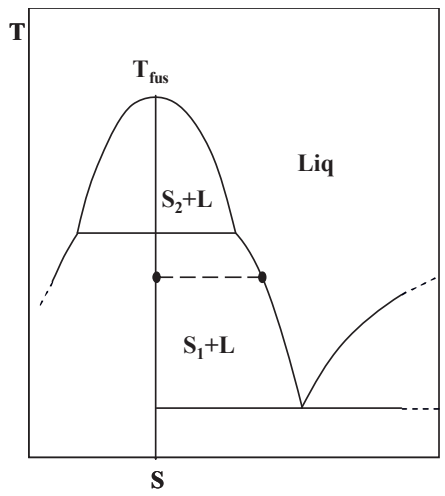

(d)

Fig. 2. Different cases of solid-liquid equilibrium in binary system. (a) congruent melting of stoichiometric coumpound (b) congruent melting of solid solution (c) peritectic decomposition (d) structural transition 
- this compound is not stoichiometric and its actual composition is different from its theoretical composition [3]: congruent melting is observed at $\mathrm{T}=1085^{\circ} \mathrm{C}$ for stoichiometry $\mathrm{K}_{2} \mathrm{O}-1.173 \mathrm{Y}_{2} \mathrm{O}_{3}-4 \mathrm{WO}_{3}$.

- it has a nonstoichiometric domain which extends to approximately $2 \mathrm{~mol} \%$ in $\mathrm{Y}_{2} \mathrm{O}_{3}$,

- there is structural transition at temperature $\mathrm{T}=1034^{\circ} \mathrm{C}$ close to melting, between a monoclinic low temperature form $(\alpha)$ and a quadratic high temperature form $(\beta)$. Only the low temperature phase $\alpha$ shows the desired optic properties [4].

Figure 3 shows the polythermal projection of the crystallisation domains for the two polymorphous varieties $\alpha$ and $\beta$ of the $\mathrm{KY}\left(\mathrm{WO}_{4}\right)_{2}$ compound [5]. Given the wide crystallization domain of phase $\alpha$, several liquid phases may be used to fabricate single crystals and the choice of their composition may be adapted according to the crystal growth processing conditions and the actual composition of a single crystal [6].

\subsection{Equilibrium disruption}

Equilibrium disruption is performed by changing the pressure, concentration or temperature in order to create a supersaturation of the mother phase, i.e. to keep this mother phase beyond the equilibrium point without a transition occurring. For instance, in the case of crystallisation from a melt, the mother phase is maintained liquid below its melting equilibrium temperature: this is the supercooling phenomenon.

Supersaturation is defined by the chemical potential difference of the constituent $i$ between the mother phase which is maintained in metastable equilibrium and the new ordered phase

$$
\Delta \mu_{i}=\mu_{i, \text { mother phase }}-\mu_{\mathrm{i}, \text { new phase }}
$$

This supersaturation may be easily expressed during a change in isothermal concentration when working from a solution or during a change in isobaric temperature when considering the simple model of ideal solution for the "mother" phase, and when the condensed phases are incompressible.

\subsubsection{Supersaturation by concentration change}

In the case of crystalline growth from solution, the supersaturation will correspond to the increase in mother solution concentration $C$ compared to the solubility equilibrium concentration $C_{e}$ at temperature $T$. The chemical potential of the supersaturated mother solution is then expressed as a function of the concentration ratio.

$$
\mu_{\text {solution }} \cong \mu_{e}+k T \ln \frac{c}{c_{e}}
$$

where $\mu_{e}$ is the chemical potential at equilibrium and $k$ is Boltzmann's constant.

If we consider that the chemical potential of crystal $\mu_{\text {crystal }}$ does not change compared to the stable equilibrium

$\mu_{\text {crystal }} \cong \mu_{e}$

Relation (3) then becomes

$$
\Delta \mu=\mu_{\text {solution }}-\mu_{\text {crystal }}=k T \ln \frac{c}{c_{e}}
$$

\subsubsection{Supersaturation by temperature change}

When crystal growth is performed from the melted compound, usually under atmospheric pressure, supersaturation is obtained at a temperature $T$ lower than the crystallisation (or fusion) equilibrium temperature $T_{e}$. The mother phase and ordered phase chemical potentials are then respectively expressed according to the temperature gap per relations (7) and (8) in which $S_{\text {liq }}$ and $S_{\text {crystal }}$ represent the molar entropy of each phase:

$$
\begin{aligned}
& \mu_{\text {liq }} \cong \mu_{e}+S_{\text {liq }}\left(T_{e}-T\right) \\
& \mu_{\text {crystal }} \cong \mu_{e}+S_{\text {crystal }}\left(T_{e}-T\right)
\end{aligned}
$$

Considering the constant entropy on a narrow temperature domain, supersaturation is then given by relation (9) in which the entropy change is regarded as melting entropy. The temperature gap, always positive, is called supercooling.

$$
\Delta \mu=\mu_{\text {liq }}-\mu_{\text {crystal }}=\Delta S_{\text {fusion }}\left(T_{e}-T\right)
$$

\section{Nucleation}

Disturbance of thermodynamic equilibrium is not enough to explain crystal creation. For instance, a liquid phase may be kept for a long time at a temperature $\mathrm{T}$ lower than $\mathrm{T}_{\text {fusion; }}$; this phase is metastable and it will only take a shock to observe spontaneous crystallisation corresponding to a return to equilibrium. Forming a new phase in the metastable mother phase will then require a nucleation stage followed by a growth. Supersaturation will locally lead to the rationalisation of entities and the formation of seeds or clusters which form the crystalline lattice. It is only from these seeds, i.e. entities of sufficient size grouping together, that crystals will be able to grow according to variable growth kinetics.

Nucleation has two origins: a) thermal agitation produces random local atom (or molecule) arrangements in the mother phase - this is homogenous nucleation; b) foreign particles suspended in the medium or a surface (substrate) will "catalyse" a new phase nucleation - this is heterogeneous nucleation. 
The energy variation that goes with the creation of a nucleus results from two factors [7-9]:

- a decrease of free energy due to the transition of the disordered mother phase into an ordered phase ;

- an energy increase associated with the creation of the interface between the two phases.

For a spherical nucleus of radius $r$ formed by homogeneous nucleation, the energy balance relative to the volume unit is expressed by relation (10) where $\sigma$ is the surface free energy of the nucleus/liquid interface.

$\Delta G_{V}=-\frac{4}{3} \pi r^{3} \Delta \mu+4 \pi r^{2} \sigma$

Whatever the nucleus geometry, the evolution of this energy according to nucleus size will go through a maximum that will correspond to the critical size from which the seed will be stable and able to grow. As an example, Table 2 compares the energy balance to create a spherical seed with a radius of curvature $r$ in the case of homogeneous and heterogeneous nucleation. The variation $\Delta \mu$ may be regarded as the difference between the free enthalpies of the phases measured on macroscopic samples, i.e. $\Delta \mathrm{G}_{\text {fusion }}$ (or $-\Delta \mathrm{G}_{\text {crystallisation }}$ ) in the case of a solid-liquid transition.

\section{Conclusion}

The thermodynamic approach to crystalline growth provides an essential theoretical basis for any singlecrystal fabrication process. This is all the more important as desired matrices become more and more complex and the homogeneity of the material's chemical composition conditions its process through the structure/property relationship. Knowledge of the relations between phases is an essential prerequisite to choosing the crystal growth technique. The most widely used industrial techniques for massive single crystals include:

- rapid growth techniques (a few $\mathrm{mm} \cdot \mathrm{h}^{-1}$ ) such as Verneuil, Czochralski, Bridgman, which are characterised by a crystallisation directed from a melt and are totally adapted to congruent melting compounds for which the melted liquid has the same composition as the crystallised solid;

- slow growth techniques (a few mm.day ${ }^{-1}$ ) which are solution or flux growths; they usually operate at a lower temperature and are used for non-congruent melting crystals (liquid and solid do not have the same composition) or those with a structural transition.
Thermodynamic considerations also involve the dependence of the supersaturation phenomena with nucleation energy and seed size that will result in the irreversible growth of macroscopic entities.

Table 2. Comparison of homogenous nucleation / heterogeneous nucleation for the critical radiuses and energy barriers.

\begin{tabular}{|l|}
\hline Homogeneous nucleation \\
\hline$r^{*}=-\frac{2 \sigma}{\Delta G_{\text {crist. }}} \quad ;$ \\
$\sigma:$ surface free energy of the interface \\
\hline Heterogeneous nucleation \\
$r^{*}=\frac{16 \pi \sigma^{3}}{3 \Delta G_{\text {crist }}^{2}}$ \\
$\Delta G^{*}=\frac{16 \pi \sigma^{3}}{3 \Delta G_{\text {crist }}{ }^{2}}\left[\frac{(1-\cos \theta)^{2}(2+\cos \theta)}{4}\right]$ \\
$\sigma:$ surface free energy of the interface \\
$\theta:$ wetting angle
\end{tabular}

\section{References}

1. H.J. Scheel, J. Crystal Growth, 211, 1-12 (2000)

2. D.J.T. Hurle, Handbook of Crystal Growth, vol. 1, (Elsevier, Amsterdam)

3. E. Gallucci, C. Goutaudier, G. Boulon, M.T. CohenAdad, Eur. J. of Solid State Inorg. Chem., 35, 433445 (1998)

4. S.V. Borisov, R.F. Kletsova, Sov. Phys. Cryst., 13(3), 420 (1968)

5. C. Goutaudier, E. Gallucci, G. Boulon, M.T. CohenAdad, J. Phase Equilibria, 22(3), 208-213 (2001)

6. E. Gallucci, C. Goutaudier, G. Boulon, M.T. CohenAdad, B.F. Mentzen, J. Cryst. Growth, 209, 895-905 (2000)

7. D. Turnbull, Solid State Physics, vol.3 (Academic Press, New York, 1956)

8. C. Herring, Structure and properties of solid surfaces ( R. Gomer, C.S. Smith Eds, 1969)

9. J. Burke, Cinétique de changements de phase dans les métaux (Masson \& Cie, Paris, 1968 


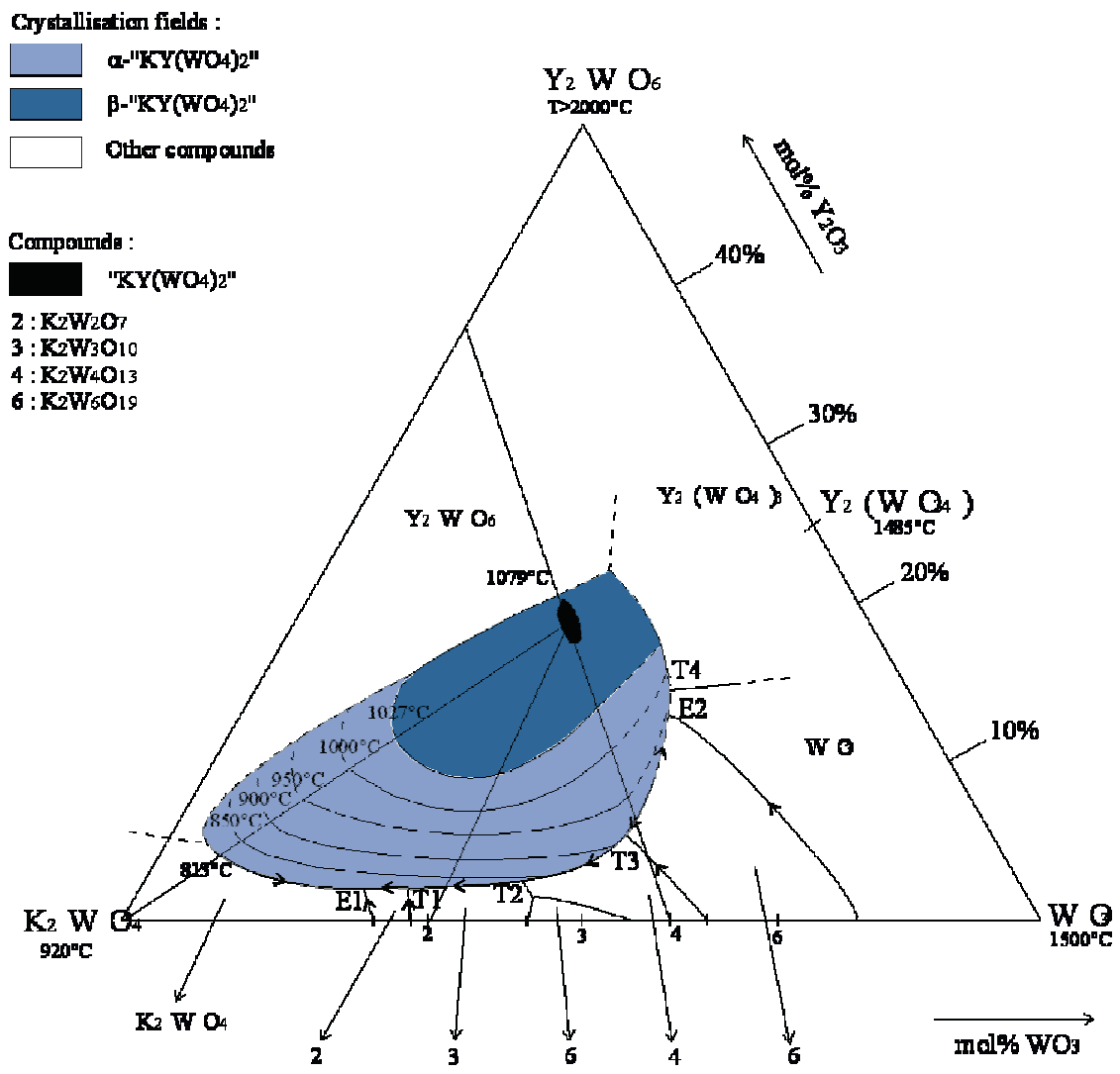

Fig. 3. Crystallization fields of the $\alpha$ - and $\beta-\mathrm{KY}\left(\mathrm{WO}_{4}\right)_{2}$ phases in the ternary sub-system $\mathrm{K}_{2} \mathrm{WO}_{4}-\mathrm{Y}_{2} \mathrm{WO}_{6}-\mathrm{WO}_{3}$ 